\title{
Development of surface oxidized metal fiber/ piezoelectric ceramics/aluminum composite
}

\author{
Kazuki HORIKIRI*, Tetsuro YANASEKO**, Isao KUBOKI**, Hiroshi SATO*** \\ and Hiroshi ASANUMA**** \\ *Graduate School of Kogakuin University \\ 2665-1 Nakanomachi, Hachioji-shi, Tokyo 192-0015, Japan \\ ** Department of Mechanical Engineering, Kogakuin University \\ 2665-1 Nakanomachi, Hachioji-shi, Tokyo 192-0015, Japan \\ E-mail: yanaseko@cc.kogakuin.as.jp \\ ${ }^{* * *}$ Advanced Manufacturing Research Institute, National Institute of Advanced Industrial Science and Technology \\ 1-2-1 Namiki, Tsukuba-shi, Ibaraki 305-8564, Japan \\ ****Department of Mechanical Engineering, Chiba University \\ 1-33 Yayoi-cho, Inage-ku, Chiba-shi, Chiba 263-8522, Japan
}

Received: 18 December 2018; Revised: 6 February 2019; Accepted: 17 February 2019

\begin{abstract}
This paper describes the fabrication of a metal matrix piezoelectric composite using surface-oxidized nickel fiber as the internal electrode. Piezoelectric ceramics, which have excellent piezoelectric properties, are widely used as energy conversion materials. However, their application is limited by their brittleness. To solve this problem, a metal-core piezoelectric ceramic fiber/aluminum composite has been developed by using the interphase forming/bonding method. Here, a piezoelectric ceramic is reinforced by embedding it in an aluminum matrix, and this process causes the piezoelectric ceramic to have better mechanical properties than a bulk ceramic. However, this composite has some serious disadvantages considering that it cannot be designed to possess arbitrary piezoelectric properties because the metal-core piezoelectric fiber is formed by the extrusion method, so that the sectional shape cannot be arbitrarily changed. In this paper, a metal matrix piezoelectric composite using a surface-oxidized nickel fiber as the internal electrode is proposed. This composite provides design flexibility in that its piezoelectric property can be changed by varying the size and materials of the composite. The fabrication procedure of this composite consists of three steps: oxidation of the internal electrode, molding and sintering of the piezoelectric ceramic, and embedding of the piezoelectric ceramic into the metal matrix. The proposed composite is fabricated under optimized oxidizing and molding conditions. An impact test is performed on the fabricated composite, and the output voltage is measured. The test results indicate that the composite is capable of generating piezoelectricity. Overall, the study results substantiate the validity of the concept and fabricating method of the proposed composite.
\end{abstract}

Keywords : Smart materials, Composites, Energy conversion, Aluminum alloy

\section{Introduction}

In recent years, there has been considerable interest in the concept and applications of the Internet of Things (IoT). In Japan, where a large number of earthquakes occur every year, IoT-based solutions are being actively researched for the problems caused by the earthquakes. Particularly, one of these solutions, structural health monitoring (SHM) based on IoT, has attracted considerable attention (Aktan et al., 1998; Brownjohn, 2007). SHM requires sensors for detecting problems in structures. There are several types of sensors such as piezoelectric type (Duan et al., 2010), optical fiber (Cusano et al., 2006), and strain gauge type (Laflamme et al, 2012) and piezoelectric sensors are very suitable for this purpose because they do not require an energy source considering that they produce piezoelectricity by themselves. However, piezoelectric ceramics, which are the most commonly used piezoelectric materials, are brittle (Mehta et al., 
1990; Yoon et al., 1997). Therefore, it is difficult to use piezoelectric ceramics in areas experiencing large strain. In previous studies, a metal-core piezoelectric fiber/aluminum composite (Asanuma et al., 2006; Asanuma et al., 2011) was developed by embedding a metal-core piezoelectric fiber (Sato et al., 2003; Qiu et al., 2004; Sato et al., 2004; Sato et al., 2005) in an aluminum matrix by the interphase forming/bonding (IF/B) method (Asanuma, 2000). This composite can generate piezoelectricity even when the strain increases to more than twice the fracture strain of bare ceramics. However, this composite has a disadvantage as a sensor - it is difficult to design the composite such that it possesses arbitrary piezoelectric properties because the metal-core piezoelectric fiber is formed by the extrusion method, so that the sectional shape cannot be arbitrarily changed. Therefore, it is impossible not to form a piezoelectric body on a part of the core metal fiber as the cross-sectional shape is constant and since it is extrusion molding, the material system is also limited from the viewpoint of securing fluidity of the PZT powder added binders. Moreover, it is a product of a private company (Nagamine Manufacturing Co., Ltd.) and difficult to redesign. For example, the output voltage of this composite depends on the direction of the applied strain because of the shape of the piezoelectric ceramic, which cannot be redesigned (Yanaseko et al., 2014; Yanaseko et al., 2015a and 2015b). Considering the practical application to SHM, it is necessary to realize sensor characteristics according to the application. In this composite, it is impossible to change the electrode structure due to the use of the metal core piezoelectric fiber, whose geometry is determined, that is, the sensor characteristics cannot be designed.

To overcome the abovementioned problem, in this study, a metal matrix piezoelectric composite using a surfaceoxidized nickel fiber as the internal electrode was proposed. This modified composite can be designed to possess arbitrary piezoelectric properties. The fabricating conditions was investigated. The effectiveness of the fabricating method and the concept of the composite was evaluated.

\section{Concept of proposed composite}

The schematic of the proposed composite is shown in Fig. 1. The previous composite did not provide design flexibility because a metal-core piezoelectric fiber was used as the piezoelectric ceramic and internal electrode. The proposed composite can be designed to possess arbitrary piezoelectric properties by changing the shape and material of the internal electrode, piezoelectric ceramic, and metal matrix. The internal electrode is coated with an oxide film, which prevents a chemical reaction from occurring between the electrode and piezoelectric ceramic. In the previous composite, the piezoelectric ceramic was exposed at the ends of the matrix, as shown in Fig. 2. In the proposed composite, the piezoelectric ceramic is embedded completely in the metal matrix. Hence, the internal electrode is contiguous with the metal matrix. Metal matrix and internal electrode are used as electrodes and have to be insulated from each other. Therefore, the oxide film, which is coated on the internal electrode, plays the important role of an insulator.

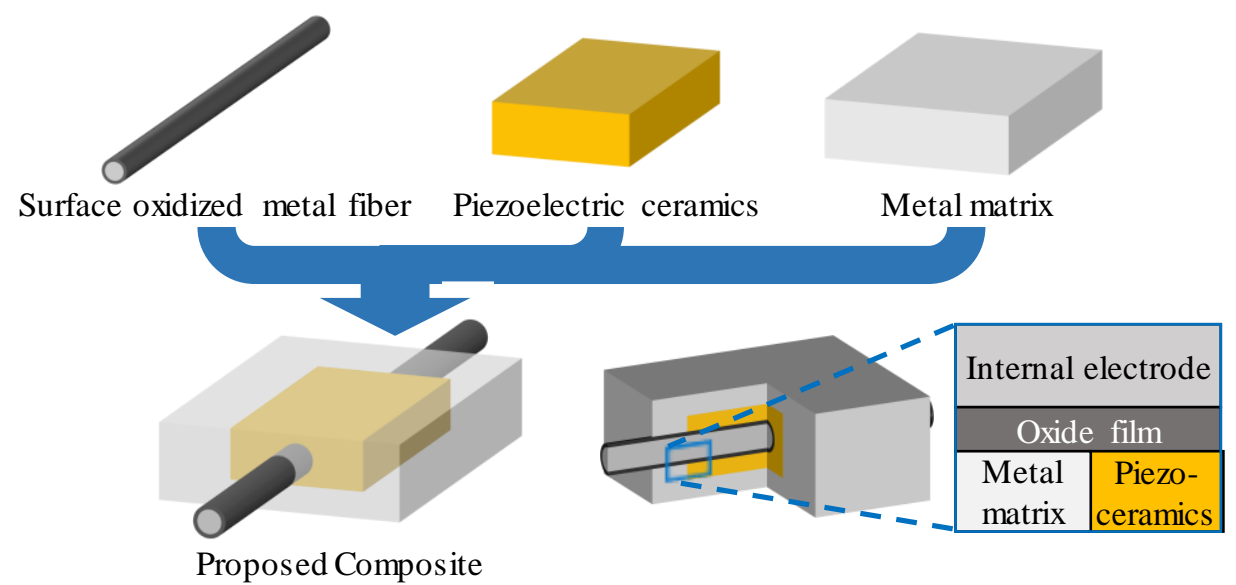

Fig. 1 Schematic of proposed metal matrix piezoelectric composite. Using surface oxidized metal fibers for the internal electrode, insulation between the internal electrode and the matrix is realized. 


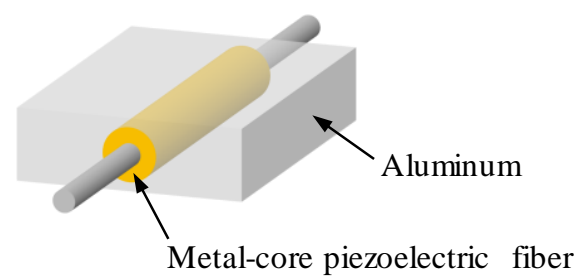

(a) Schematic

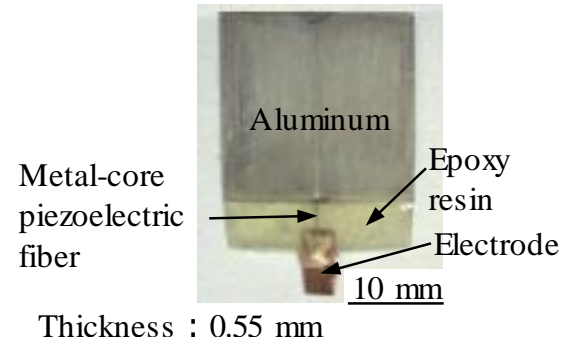

(b) General appearance

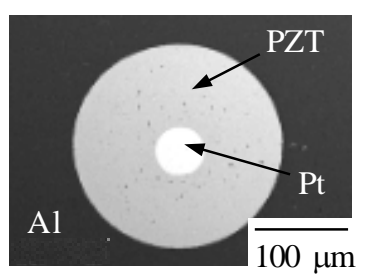

(c) Microstructure

Fig. 2 Metal-core piezoelectric fiber/aluminum composite; (a) schematic, (b) general appearance and (c) microstructure. Since the metal-core piezoelectric fiber is used, it is impossible to change the electrode structure.

\section{Experimental methods 3.1 Material selection}

To realize the proposed composite, the material of the internal electrode was selected. The internal electrode material should possess the following characteristics;

1) Have a higher melting point than the sintering temperature of the piezoelectric ceramic;

2) Be suitable for coating with a fine oxide film;

3) Be inexpensive.

Nickel matched to these requirements. Therefore, nickel was selected as the internal electrode.

The piezoelectric ceramic used in this study is lead zirconate titanate (PZT), which has excellent piezoelectric properties and is the most commonly used piezoelectric ceramic. Its typical sintering temperature is approximately 1300 $\mathrm{K}$. Hence, based on the abovementioned requirements, nickel was selected as the internal electrode material.

The main objective of this study was to check whether the proposed composite could be realized using a surfaceoxidized internal electrode. Therefore, PZT and aluminum were selected as the materials for the piezoelectric ceramic and metal matrix based on the materials used in previous composites.

\subsection{Oxidizing of the internal electrode}

The effect of the oxidizing conditions on the thickness of the oxide film was evaluated in order to get ensure that the internal electrode could be coated with obtain an oxide film of arbitrary thickness. Pure nickel fiber (Nilaco Corporation, purity: $99.9 \%$, diameter: $0.5 \mathrm{~mm}$ ) was used as the internal electrode material. The nickel fiber was cut into $40 \mathrm{~mm}$ pieces and then oxidized by heating in the air by a muffle furnace. The thickness of the oxide film was measured by observing the cutting section using a scanning electron microscope (SEM) under various heating conditions (heating temperatures: $1073,1173,1273$, and $1373 \mathrm{~K}$, holding times: $0.9,1.8,3.6,7.2$, and $10.8 \mathrm{ks}$ ).

\subsection{Molding of PZT powder around the internal electrode}

PZT powder (Hayashi Chemical Industry Co. Ltd., HIZIRCO PZT series, MPT, average diameter: less than $0.1 \mu \mathrm{m}$ ) was sintered around the internal electrode after molding by using a uniaxial press. The effect of molding condition on sintered compact was evaluated by measuring Vickers hardness (Load: 1.96 N) and density. The PZT powder was mixed with a binder solution and then dried at $353 \mathrm{~K}$ for $7.2 \mathrm{ks}$, milled, and sieved (screen size: $100 \mu \mathrm{m}$ ). The binder solution was prepared by mixing distillated water: $200 \mathrm{~g}$, polyvinyl alcohol: $2 \mathrm{~g}$, ethanol: $24 \mathrm{~g}$, and glycerin: $0.6 \mathrm{~g}$. The sieved powder was filled in a die and uniaxially pressed under various conditions (pressure: 20-90 MPa in 10-MPa increments, holding time: $60 \mathrm{~s}$ ). The powder was then sintered in a muffle furnace under the sintering condition recommended by the supplier (sintering temperature: $1323 \mathrm{~K}$, holding time: $7.2 \mathrm{ks}$, in the air). Before sintering, the pressurized powder was degreased by holding it at $623 \mathrm{~K}$ for $18 \mathrm{ks}$.

Moreover, to confirm the effect of prevention of reaction between nickel and PZT during sintering by oxide film, cross section of sintered compact was observed by SEM and energy dispersive X-ray spectrometry (EDS) analysis was 
carried out. For comparison, a sintered compact was fabricated without the oxidation of the nickel fiber (hereinafter referred to the non-oxidized fiber). The oxide film thickness was measured by cross sectional of sintered compact by SEM observation and the consistency with the thermal oxidation treatment performed in the previous section was examined.

\subsection{Fabrication of the composite}

To verify the effectiveness of the proposed composite, the composite was fabricated by IF/B method. Aluminum (A1050P-O, thickness: 0.8 and $2.5 \mathrm{~mm}$ ) was used as metal matrix and pure copper foil (Nilaco Corporation, purity: 99.9\%, thickness: $10 \mu \mathrm{m}$ ) was used as inserts. PZT powder was molded and sintered around the surface-oxidized nickel fiber in the air. The molding condition which is chosen considering the result of the section 3.3 is used. The sintering condition is same as one in the section 3.3. The sintered compact and recesses for the piezoelectric ceramics were machined as shown in Fig. 3. The sintered compact, aluminum matrix, and insert material for the IF/B method were cut and arranged as shown in Fig. 3 and were then hot-pressed under the condition followed in previous studies (vacuum: $0.1 \mathrm{kPa}$, pressure: $2.2 \mathrm{MPa}$, temperature: $873 \mathrm{~K}$, holding time: $2.4 \mathrm{ks}$ ).

\subsection{Impact test}

An impact test was performed on the fabricated composite to verify its capability for insulation. Some parts of the oxide film coated on the nickel fiber were exposed outside the aluminum matrix; these parts were removed using abrasive paper. Then, the composite was polarized by an applied voltage of $600 \mathrm{~V}$ for $1.8 \mathrm{ks}$ between the aluminum matrix and internal electrode. The impact test system is shown in Fig. 4. The fabricated composite was fixed on a vise, and a $3.5 \mathrm{~g}$ steel ball was made to free fall on the composite from a height of $150 \mathrm{~mm}$ through a guide pipe. The output voltage of the composite was measured using an oscilloscope (Input impedance: $10 \mathrm{M} \Omega$ ).

\subsection{Observation of microstructure of the fabricated composite}

To verify the validity of the oxide film of the internal electrode, observation of microstructure of the composite was carried out. The observation site was set at two places where the nickel fiber is in contact with the matrix and where the PZT is in contact with the matrix. In addition, in order to confirm the chemical reaction between piezoelectric ceramics and matrix by embedding, elemental analysis by EDS was carried out.

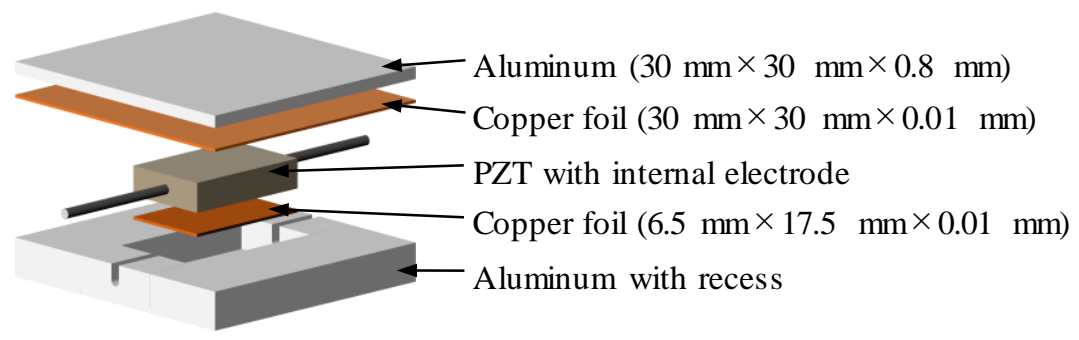

(a) Arrangement of aluminum, PZT and inserts

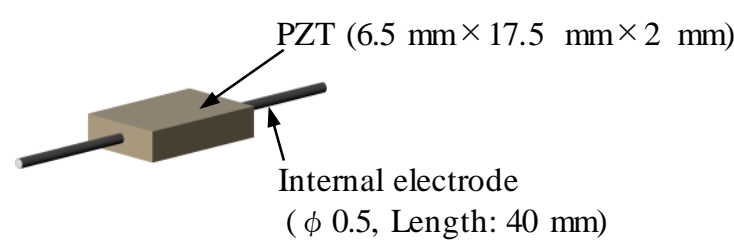

(b) PZT with internal electrode

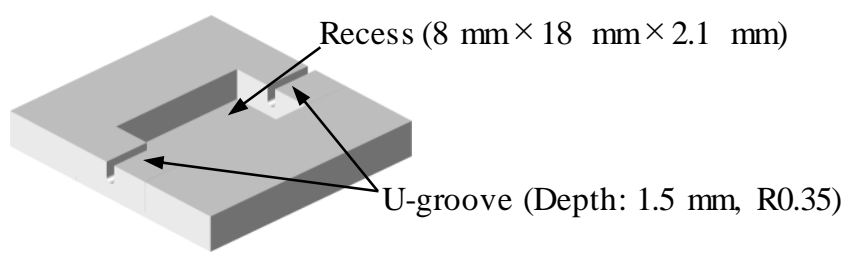

(c) Aluminum with recess

Fig. 3 Arrangement and shapes of aluminum, PZT with electrode and copper inserts. PZT is grinded and polished as shown in (b) after sintering, and the recess is machined as shown in (c). After that, they were arranged as shown in (a) and hot pressed. 


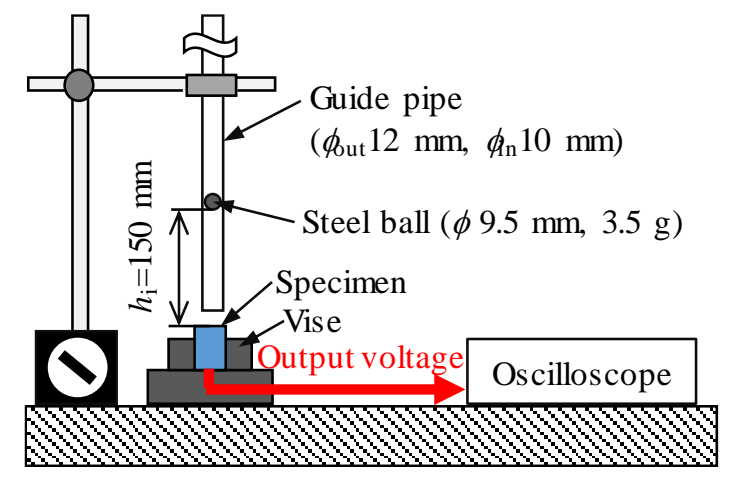

Fig. 4 Schematic of impact test system. By applying an impact and measuring the voltage generated from the specimen, it was confirmed whether the piezoelectric properties of PZT were embedded in aluminum without losing its function.

\section{Results and discussion}

\subsection{Oxidizing of the internal electrode}

The thickness of oxide film of the nickel fiber was measured by observing the cutting section using SEM. Figure 5 shows the effect of square root of holding time on the thickness of the oxide film of the nickel fiber. According to this result, the thickness of the oxide film is proportional to the square root of holding time in each temperature. When the metal is heated to high temperature in air, an oxide film is formed on the surface. If this oxide film covers the surface densely and uniformly, the subsequent reaction will cause mass transport through this film (Out diffusion of metal and inward diffusion of oxygen). Therefore, the growth rate (oxidation rate) of the oxide film will be inversely proportional to the thickness of the film, and it will be written as in Eq. (1),

$$
\frac{\mathrm{d} X}{\mathrm{~d} t}=\frac{k}{X}
$$

Here, $X$ is thickness of oxide film, $k$ is oxide reaction rate and $t$ is time.

Equation (2) is obtained by integrating Eq (1), that is, parabolic law, and the experimental result agrees well with this equation.

$$
X^{2}=k t
$$

Figure 6 shows the Arrhenius plot of proportionality. All the plotted points can be approximated by a straight line. Therefore, the thickness of the oxide film can be estimated by this line and the Arrhenius equation. From these results, it is found that a dense and uniform oxide film is obtained, which is a single generation mechanism.

In this study, the fabricated composite was polarized by $600 \mathrm{~V}$. The breakdown voltage of nickel oxide is about 50 to $800 \mathrm{~V} / \mu \mathrm{m}$ (Krishnan et al., 2007 and Yoo et al., 2008), and when a minimum value of $50 \mathrm{~V} / \mu \mathrm{m}$ is used, the film thickness necessary for insulation is $10 \mu \mathrm{m}$. It is known that breakdown voltage of oxide film decreases due to defects in itself and breakdown voltage follows Weibull distribution (Yamashita et al., 1990). The literature value is the value in the thin film, and the ratio with the present oxide film is about $10^{4}$. Assuming that the effective volume is simply proportional to the film thickness, the insulation voltage was corrected by Eq. (3).

$$
\frac{E_{a}}{E_{l}}=\left(\frac{V_{l}}{V_{a}}\right)^{\frac{1}{m}}
$$

Here, $m$ is Weibull modulus, the actual breakdown voltage and effective volume are $E_{\mathrm{a}}, V_{\mathrm{a}}$, and the breakdown voltage and effective volume in the literature are $E_{1}, V_{\mathrm{l}}$, respectively. And $m$ was set to 15 ( $m$ of common ceramics: 10 to 20 (Danzer et al., 2007, Nakamura et al., 2009 and Quinn et al., 2010), middle values are used). 


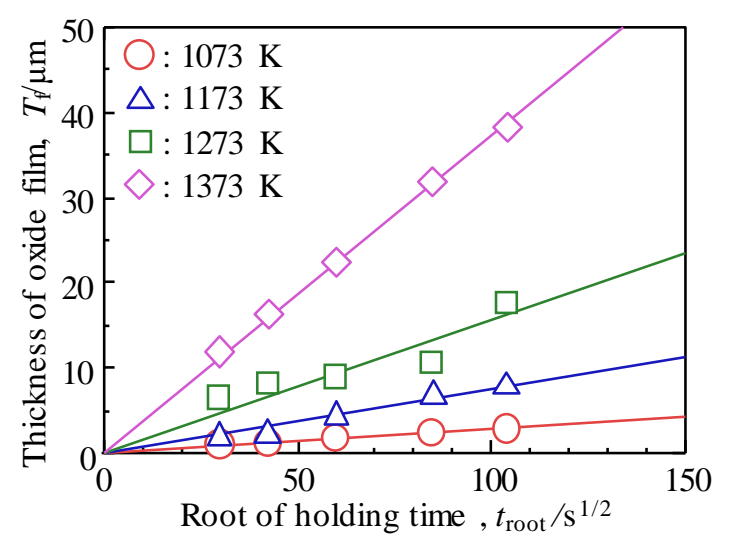

Fig. 5 Effect of square root of holding time on thickness of oxide film. The thickness is proportional to root of holding time at each temperature.

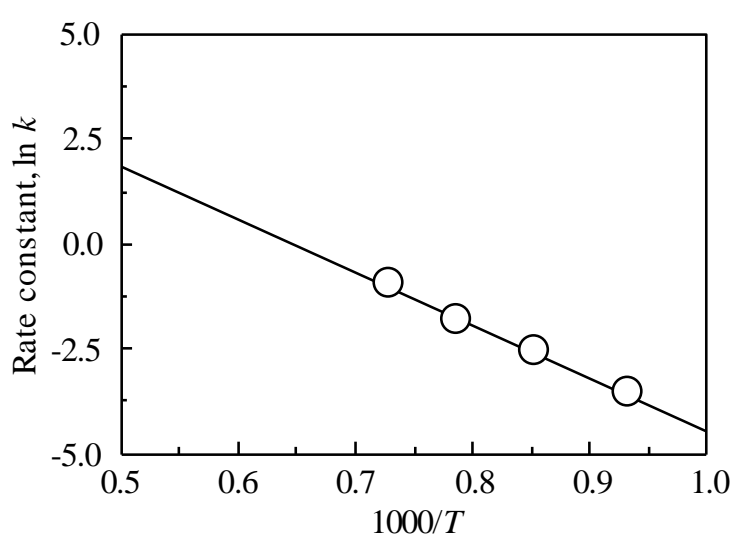

Fig. 6 Temperature dependence of oxidation rate constant. It is linear to the reciprocal of temperature and consistent with Arrhenius's equation

The safety factor when using the literature value can be calculated by Eq. (3), and its value is 1.84 . For this value, the safety factor was set to 2 , and it was judged that insulation was possible if an oxide film of $20 \mu \mathrm{m}$ or more was produced. Assuming that oxygen is sufficiently supplied and an oxide film grows according to Fig. 5 and Fig. 6 even during sintering (sintering temperature: $1323 \mathrm{~K}$, holding time: $7.2 \mathrm{ks}$, in the air ), it can be estimated that it grows about $15 \mu \mathrm{m}$. Since the insulation is insufficient with film thickness of $15 \mu \mathrm{m}$, the oxide film is formed by heat treatment beforehand. The conditions of the heat treatment are temperature of $1273 \mathrm{~K}$ for $7.2 \mathrm{ks}$, and the film thickness which grows at this case is about $10 \mu \mathrm{m}$. This is a condition that anticipates safety and secures a large film thickness (about 25 $\mu \mathrm{m})$.

\subsection{Molding of PZT powder around the internal electrode}

Figure 7 shows the effect of pressure on Vickers hardness and density of the sintered compact. From 20 to $50 \mathrm{MPa}$, as the pressure increase, both hardness and density increase. From 50 to $90 \mathrm{MPa}$, the amounts of them seem to be saturated. This suggests that the density of the PZT green compact increased due to an increase in pressure, and the closest powder compact was obtained at $50 \mathrm{MPa}$. In addition, at more than $50 \mathrm{MPa}$, it is considered that the density of the green compact has saturated because it is already closest. According to this result, $50 \mathrm{MPa}$ was selected as molding pressure.

Figure 8 shows SEM images of cross section and the result of element analysis by EDS. According to this figure, the thickness of the oxide film was (a) $25.8 \mu \mathrm{m}$ and (b) $17.1 \mu \mathrm{m}$. This is in good agreement with the respective film thickness derived in section 4.1. From this result, it is assumed that (a)oxidized fiber is supplied with oxygen by diffusing oxygen

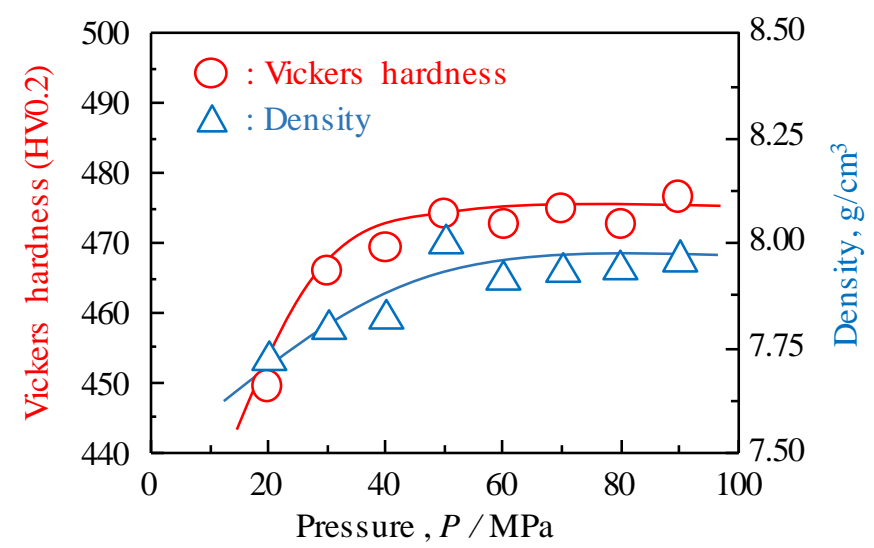

Fig. 7 Effect of molding pressure on Vickers hardness and density of sintered compact. Hardness and density are improved up to $50 \mathrm{MPa}$ and almost saturated thereafter. 

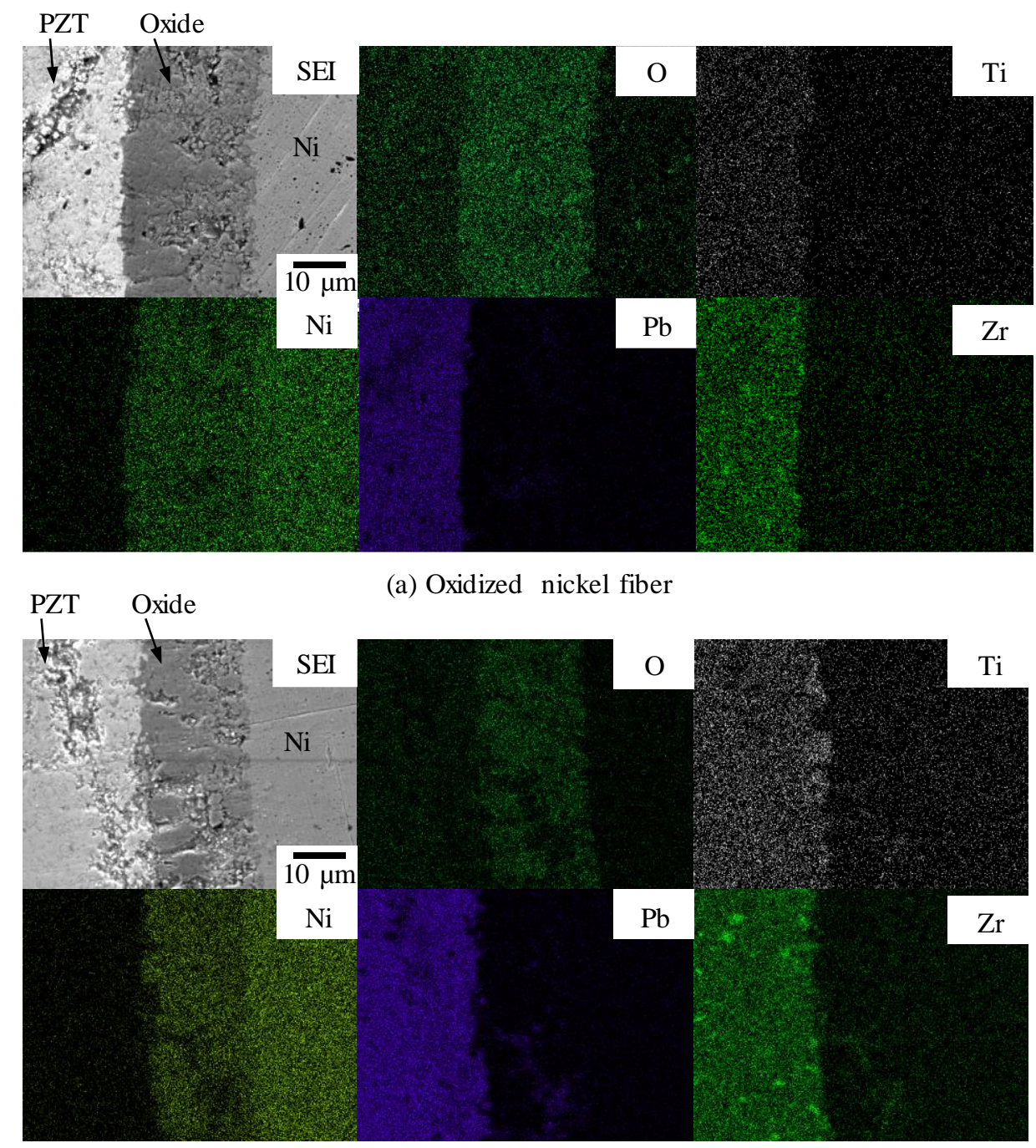

(b) Non-oxidized nickel fiber

Fig. 8 Results of elemental analysis of sintered compact (a) using oxidized (1273 K, $7.2 \mathrm{ks}$, in the air) nickel fiber and (b) using non oxidized nickel fiber. Compared with (a) and (b), it is found that the oxygen concentration in PZT of (b) is lower than that of (a). This decrease in oxygen concentration is due to the reduction of PZT by non-oxidized nickel fiber.

in the already formed oxide film and PZT. However, focusing to the oxygen distribution in the PZT part in this figure, it is found that the oxygen of (b) the non-oxidizing fiber is extremely lower than that of (a) the oxidized fiber. This is because nickel deprived oxygen of PZT and reduced. Thus, (b) non-oxidized fiber, it is suggested that nickel first reduces PZT and become oxide, then grows with oxygen passing through the oxide film. Summarizing these results, it was indicated that by forming the oxide film beforehand, the oxide film becomes path that through oxygen, so that chemical reaction between nickel and PZT can be prevented.

\subsection{Impact test}

This research focused on verifying the effectiveness of the concept of this composite. Figure 9 shows the output waveform of the composite. It was confirmed that the composite generated the electricity as the output for the impact. According to this result, it was shown that PZT embedded in aluminum matrix did not lose piezoelectricity, and this composite works as a piezoelectric sensor. However, this output voltage is about $5 \mathrm{mV}$ (peak to peak), significantly low as piezoelectric sensor. It is considered that the electrode structure and the PZT shape are not optimized with respect to the output voltage. The polarization condition is not also optimized and the residual polarization of the PZT is 


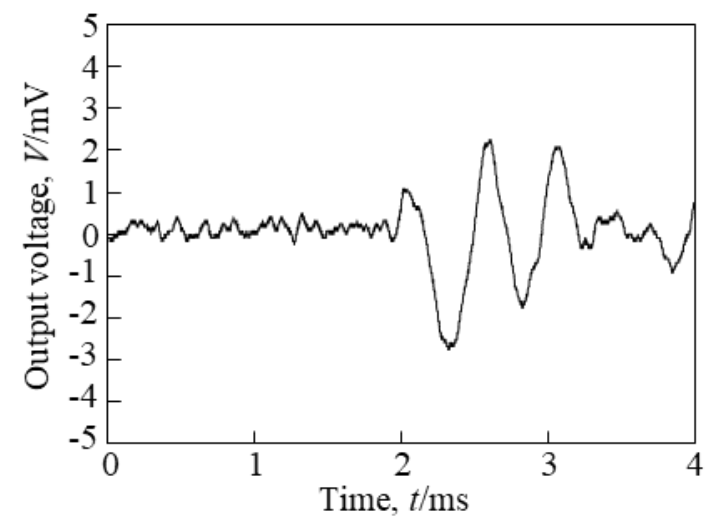

Fig. 9 Waveform of output voltage by applying an impact. From this result, embedded PZT does not lose piezoelectricity. However, output voltage is about $5 \mathrm{mV}$ (peak to peak), it is very small as piezoelectric sensor.

extremely small. Moreover, to pole the PZT, voltage of $600 \mathrm{~V}$ was applied between the nickel fiber and the aluminum matrix, however dielectric breakdown of the oxide film did not occur. This result indicates that the oxide film thickness set in section 4.1 is sufficient, and it is possible to embed the oxide film of the fiber in aluminum matrix without fracture that lead contacting between nickel and aluminum, and losing insulation.

\subsection{Observation of microstructure of the fabricated composite}

General appearance of fabricated specimen and secondary electron images of the cross section of the composite by SEM is shown in Fig. 10. According to this figure, the embedding of piezoelectric ceramics without fracture of oxide film and PZT was verified, but defects such as void and residual eutectic alloy was confirmed. These defects occur reduction of output voltage by disturbing stress transmission between matrix and PZT (Yanaseko et al., 2018). Microstructure improvements are indispensable for improving the output voltage, and improvement of the microstructure is expected to be achieved by condition optimization of the IF/B method. And there is no chemical reaction between matrix and PZT and almost no diffusion of lead into aluminum by EDS (Fig. 11). In this figure, the aluminum concentration (blue line) in the vicinity of the interface is low is because $\mathrm{CuAl}_{2}$ generated during the eutectic reaction remains at the interface.

Based on these results, it was shown that the proposed metal matrix piezoelectric composite with sensor characteristics adjustable by designing the piezoelectric ceramics shape arbitrary can be fabricated. It is succeeding that fabrication of the composite without the cracking of oxide film and PZT, and loss of piezoelectricity by using piezoelectric ceramics with surface oxidized metal fiber and the IF/B method.

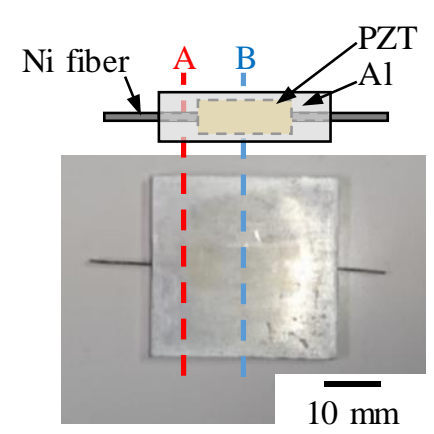

(a) Fabricated composite

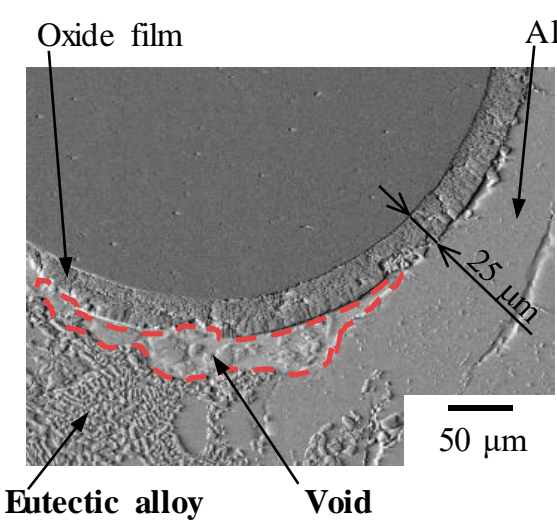

(b) Cross section A

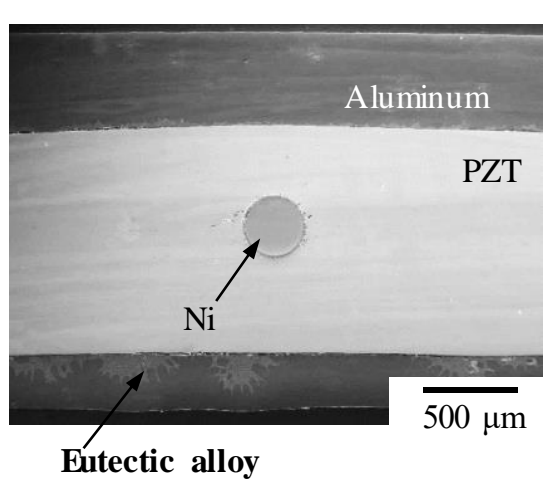

(c) Cross section B

Fig. 10 Image of fabricated composite; (a)general appearance, (b) cross section A and (c) cross section B. In (b) and (c), 
the oxide film and PZZT could be embedded without damage such as crack.

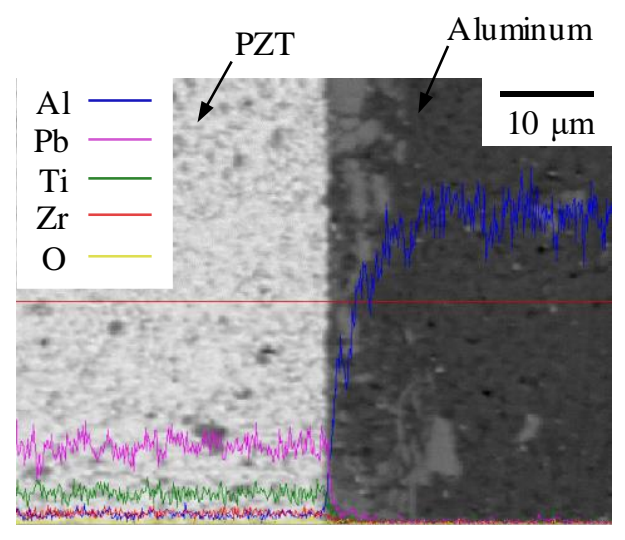

Fig. 11 Result of elemental analysis at interface of PZT/aluminum. There is no reaction product between PZT and aluminum, almost no diffusion of lead into aluminum.

\section{Conclusions}

In this study, to realize a metal-based piezoelectric composite material whose sensor characteristics can be designed by changing the shape of piezoelectric ceramics, metal matrix piezoelectric composite using piezoelectric ceramics having surface oxidized metal fiber as internal electrodes was developed by investigating fabrication condition and evaluating microstructure of composite and output voltage. Based on the study results, the following conclusions were drawn:

1) The oxide film thickness of the internal electrode can be estimated by the Arrhenius plot and equation, thus, oxide film thickness can be adjusted heat treat conditions. And the oxide film prevents the chemical reaction between nickel and PZT, this is because the oxide film becomes a path through oxygen and reduction of PZT by nickel does not occur.

2) As a result of observing the cross section of the composite, it was shown that PZT and the oxide film were compounded without cracks, and not breaking down the oxide film during the polarization and embedding the piezoelectric ceramics in aluminum without losing piezoelectricity are indicated by the impact test. Thus, the proposed fabrication method can be used to form the internal electrode, piezoelectric ceramic, and metal matrix without microstructure damage or loss of functionality. However, since it was suggested that the output voltage is as small as $5 \mathrm{mV}$, which is attributable to insufficient polarization due to the decrease of the effective polarization voltage by the oxide film, optimization of the polarization condition is necessary for higher output voltage.

3) By indicating that securing the insulation between matrix and metal fiber by oxide film, prevention of chemical reaction with piezoelectric ceramics and metal fiber, shape freedom of piezoelectric ceramics by molding, and embedding piezoelectric ceramics in metal matrix without crack using IF/B method, it was shown that the proposed metal matrix piezoelectric composite with sensor characteristics adjustable by designing the piezoelectric ceramics shape arbitrary can be fabricated.

\section{References}

Aktan, A. E., Helmicki, A. J., and Hunt, V. J., Issues in health monitoring for intelligent infrastructure, Smart Materials and Structures, 7 (1998), pp. 674-692, DOI: 10.1088/0964-1726/7/5/011.

Asanuma, H., Development of metal-based smart composites, JOM, Vol. 52, No. 10 (2000), pp. 21-25.

Asanuma, H., Takeda, N., Chiba, T., and Sato, H., Nippon Kikai Gakkai (The Japan Society of Mechanical Engineers), Collection of papers of 14th Mechanical material and material processing technology lecture meeting, (2006), pp. 21-22 (in Japanese).

Asanuma, H., Sato, H., Functional composite material equipped with embedded piezoelectric fiber with metal core, 
Japanese Patent, No. 4719897 (2011).

Brownjohn, J. M. W., Structural health monitoring of civil infrastructure, Philosophical Transactions of the Royal Society A, 365 (2007), 589-622, DOI: 10.1098/rsta.2006.1925.

Cusano, A., Capoluongo, P., Campopiano, S., Cutolo, A., Giordano, M., Felli, F., Paolozzi, A. and Caponero, M., Experimental modal analysis of an aircraft model wing by embedded fiber Bragg grating sensors, IEEE Sensors Journal, 6 (2006), pp. 37-77, DOI: 10.1109/JSEN.2005.854152.

Danzer, R., Supancic, P., Pascual, J., Lube, T., Fracture statistics of ceramics - Weibull statistics and deviations from Weibull statistics, Engineering Fracture Mechanics, 74(2007), pp. 1-20.

Duan, W. H., Wang, Q. and Quek, S. T., Applications of Piezoelectric Materials in Structural Health Monitoring and Repair: Selected Research Examples, Materials, 3(2010), pp. 5169-5194, DOI: 10.3390/ma3125169.

Krishnan, S., Stefanakos, E. and Bhansali, S., Effects of dielectric thickness and contact area on current-voltage characteristics of thin film metal-insulator-metal diodes, Thin Solid Film, 516 (2008), pp. 2244-2250. DOI:10.1016/j.tsf.2007.08.067.

Laflamme, S., Kollosche, M., Kollipara, V. D., Saleem, H. S. and Kofod G., Large-scale surface strain gauge for health monitoring of civil structures, Proceedings of SPIE, Smart Structures and Materials 2012: Nondestructive Evaluation and Health Monitoring, 2012, Paper No. 8347, DOI: 10.1117/12.913187.

Mehta, K. and Virkar, A. V., Fracture Mechanisms in Ferroelectric-Ferroelastic Lead Zirconate Titanate $(\mathrm{Zr}: \mathrm{Ti}=0.54: 0.46)$ Ceramics, Journal of the American Ceramics Society, Vol. 73, Issue 3 (1990), pp. 567-574.

Nakamura, S., Tanaka, S., Furushima, R., Sato, K., Umetsu, K., Estimation of Weibull modulus from coarser defect distribution in dry-pressed alumina ceramics, Journal of the Ceramic Society of Japan, 117, 6(2009), pp.742-747.

Qiu, J., Tani, J., Yamada, N. and Takahashi, H., Fabrication of piezoelectric fibers with metal core, Proceedings of SPIE, Smart Structures and Materials 2003: Active Materials: Behavior and Mechanics (2003), Paper No. 5053, DOI: $10.1117 / 12.484355$.

Quinn, J. D., Quinn, J. B., A practical and systematic review of Weibull statistics for reporting strengths of dental materials, Dental Materials, 26, 2(2010), pp. 135-147.

Sato, H., Shimojo, Y. and Sekiya, T., Development of the smart board using metal core piezoelectric complex fibers, Proceedings of TRANSDUCERS, Solid-State Sensors, Actuators and Microsystems, 12th International Conference on, 2003, DOI: 10.1109/SENSOR.2003.1215366.

Sato, H., Shimojo, Y. and Sekiya, T., Lead zirconate titanate fiber, smart board using lead zirconate titanate fiber, actuator utilizing smart board, and sensor utilizing smart board, US patent, US6963157 B2 (2005).

Sato, H., Sekiya, T. and Nagamine, M., Design of the metal-core piezoelectric fiber, Proceedings of SPIE, Smart Structures and Materials 2004: Smart Structures and Integrated Systems (2004), Paper No. 5390, DOI:10.1117/12.540644.

Sato, H. and Nagamine, M., Mechanical properties of metal-core piezoelectric fiber, Proceedings of SPIE, Smart Structures and Materials 2005: Smart Structures and Integrated Systems (2005), Paper No. 5764, DOI:10.1117/12.601109.

Yamashita, K., Umegaki, T., Koumoto, K., Yanagida, H., Variation of Weibull Modulus as the Scattering Parameter for Dielectric Strength of Barium Titanate Thick Films, Journal of the Ceramic Society of Japan, 98, 8 (1990), pp. 909-912.

Yanaseko, T., Asanuma, H. and Sato, H., Output Voltage Characteristics of Piezoelectric Fiber/Aluminum Composites Fabricated by Interphase Forming/Bonding Method, Transaction of the Material Research Society of Japan, 39 (2014), pp. 325-329, DOI: 10.14723/tmrsj.39.325.

Yanaseko, T., Asanuma, H. and Sato, H., Characterization of a metal-core piezoelectric ceramics fiber/aluminum composite, Mechanical Engineering Journal, Vol. 2, No. 2 (2015a), DOI:10.1299/mej.14-00357.

Yanaseko, T., Asanuma, H. and Sato, H., Detection of Impact Location by Using Anisotropy of Output Voltage of MetalCore Piezoelectric Fiber/Aluminum Composites, International Journal of Materials Science and Applications, 4(2015b), pp. 256-260, DOI: 10.11648/j.ijmsa.20150404.15.

Yanaseko, T., Asanuma, H., Kuboki, I. and Sato, H., Effect of microstructure of metal-core piezoelectric fiber/aluminum composites on output voltage characteristics. Mechanical Engineering Journal, 5 (2018), DOI: 10.1299/mej.1700565.

Yoo, I. K., Kang, B. S., Park, Y. D., Lee, M. J., Park, Y., Interpretation of nanoscale conducting paths and their control in nickel oxide (NiO) thin films, Applied Physics Letter, 92, 202112(2008), pp. 1-3. 
Horikiri, Yanaseko, Kuboki, Sato and Asanuma, Mechanical Engineering Journal, Vol.6, No.3 (2019)

Yoon, S. J., Moon, J. H. and Kim, H. J., Piezoelectric and mechanical properties of $\mathrm{Pb}(\mathrm{Zr} 0.52, \mathrm{Ti0.48}) \mathrm{O} 3 \mathrm{~Pb}(\mathrm{Y} 2 / 3$, W1/3)O3(PZT-PYW) ceramics, Journal of Materials Science, Vol. 32, Issue 3 (1997), pp 779-782. 